\title{
Intensity Dependent Femtosecond Dynamics in a PBDTTPD-Based Solar Cell Material
}

\author{
Arun Aby Paraecattil,,$^{\dagger}$ Serge Beaupré, ${ }^{\ddagger}$ Mario Leclerc, ${ }^{\ddagger}$ Jacques-E. Moser, ${ }^{\dagger}$ and Natalie Banerji ${ }^{*}{ }^{\dagger}$ \\ ${ }^{\dagger}$ Institute of Chemical Sciences \& Engineering, Ecole Polytechnique Fédérale de Lausanne (EPFL), SB ISIC GR-MO, Station 6, \\ CH-1015 Lausanne, Switzerland \\ ${ }^{\ddagger}$ Department of Chemistry, Université Laval, G1K 7P4 Quebec City, Quebec, Canada
}

Supporting Information

\begin{abstract}
PBDTTPD is a conjugated polymer with high power conversion efficiency if used in organic solar cells together with fullerene derivatives. We have investigated for the first time the excited state dynamics of pristine PBDTTPD thin film as well as the ultrafast evolution of charge carriers in PBDTTPD:PCBM bulk heterojunction blend using femtosecond transient absorption spectroscopy. In the latter, charges appear within the time resolution of the experiment $(<100 \mathrm{fs})$, but clean spectral signatures allowed to directly follow slower $\sim 1$ ps charge separation. Only the slower quenching component competes with exciton-exciton and exciton-charge annihilation, leading to a reduced yield of charge carriers at high laser fluence. Our excellent measuring sensitivity made it possible to reduce pump power to a point where annihilation is quasi suppressed. In this case $>80 \%$ of charges survive after $1 \mathrm{~ns}$; the rest recombines (most probably geminately) on the 200 ps time scale.
\end{abstract}

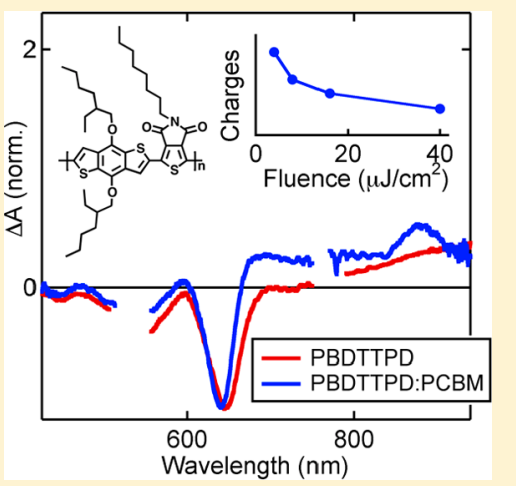

SECTION: Energy Conversion and Storage; Energy and Charge Transport

$\mathrm{C}_{\mathrm{r}}^{\mathrm{o}}$ onjugated polymers have enjoyed success in organic photovoltaic devices. They are typically blended with fullerene derivatives such as $[6,6]$-phenyl- $\mathrm{C}_{60}$-butyric acid methyl ester (PCBM), which accept an electron from the photoexcited polymer leading to the formation of charge carriers that are ultimately extracted through electrodes to yield macroscopic current. ${ }^{1,2}$ Poly(benzo[1,2-b:4,5-b'] dithiophenealt-thieno[3,4-c]pyrrole-4,6-dione (PBDTTPD), shown in the inset of Figure 1, was first reported in 2010 by several groups. ${ }^{3-5}$ By optimizing the morphology of the PBDTTPD:PCBM blend using coadditives, Leclerc et al. recently obtained $7.1 \%$ power conversion efficiency in $1 \mathrm{~cm}^{2}$ active layer devices, remarkable for solar cells containing the $\mathrm{C}_{60}$ derivative. ${ }^{6}$ PBDTTPD bears alternating benzodithiophene (BDT) electron-donating groups and thieno[3,4-c]pyrrole-4,6dione (TPD) electron-withdrawing groups along its backbone, a strategy that is commonly employed to reduce the bandgap of conjugated polymers.

The photophysics of PBDTTPD has only been little explored. ${ }^{7,8}$ We report here, to our knowledge, the first transient absorption (TA) study of the ultrafast processes occurring in thin films of pristine PBDTTPD and PBDTTPD:PCBM blend. TA spectroscopy has been used on numerous occasions to understand the charge carrier dynamics in polymer:fullerene blends. ${ }^{9-17}$ It is, however, becoming clear that the important energy delivered by pulsed laser excitation in such experiments is not necessarily representative of solar irradiation: The high density of excited species and charges generated at high pump fluence leads to bimolecular loss mechanisms that are not observed under solar cell operating conditions. ${ }^{10-12,17-20}$ Excellent measuring sensitivity of our apparatus has allowed us to reduce the excitation power to a point where those loss mechanisms become negligible on the subnanosecond time scale. Moreover, we have taken advantage of the annihilation processes that occur with increasing fluence to gain valuable insight about the evolution of excited and charged species in pristine and blended PBDTTPD.

Figure 1A depicts the steady-state absorption and fluorescence spectra of the investigated thin films. Pristine PBDTTPD has a broad and structured absorption band ranging from $\sim 360-700 \mathrm{~nm}$. The presence of defined vibronic features, uncommon for conjugated donor-acceptor copolymers, has been attributed to a planar backbone conformation of the polymer in the ground state (density functional thoery calculations), ${ }^{21}$ and to insignificant torsional relaxation in the excited state (two-dimensional electronic spectroscopy). ${ }^{8}$ The quantum simulations also suggest delocalized highest occupied molecular orbital (HOMO) and lowest unoccupied molecular orbital (LUMO) levels and that several electronic bands might be present under the broad envelope of the absorption spectrum. ${ }^{21}$ The emission spectrum of pristine PBDTTPD film is weakly Stokes shifted with a maximum at $662 \mathrm{~nm}$ and a vibronic shoulder around $720 \mathrm{~nm}$. For the $1: 2$ PBDTTPD:PCBM blend cast from $o$-dichlorobenzene, the

Received: August 3, 2012

Accepted: September 25, 2012 


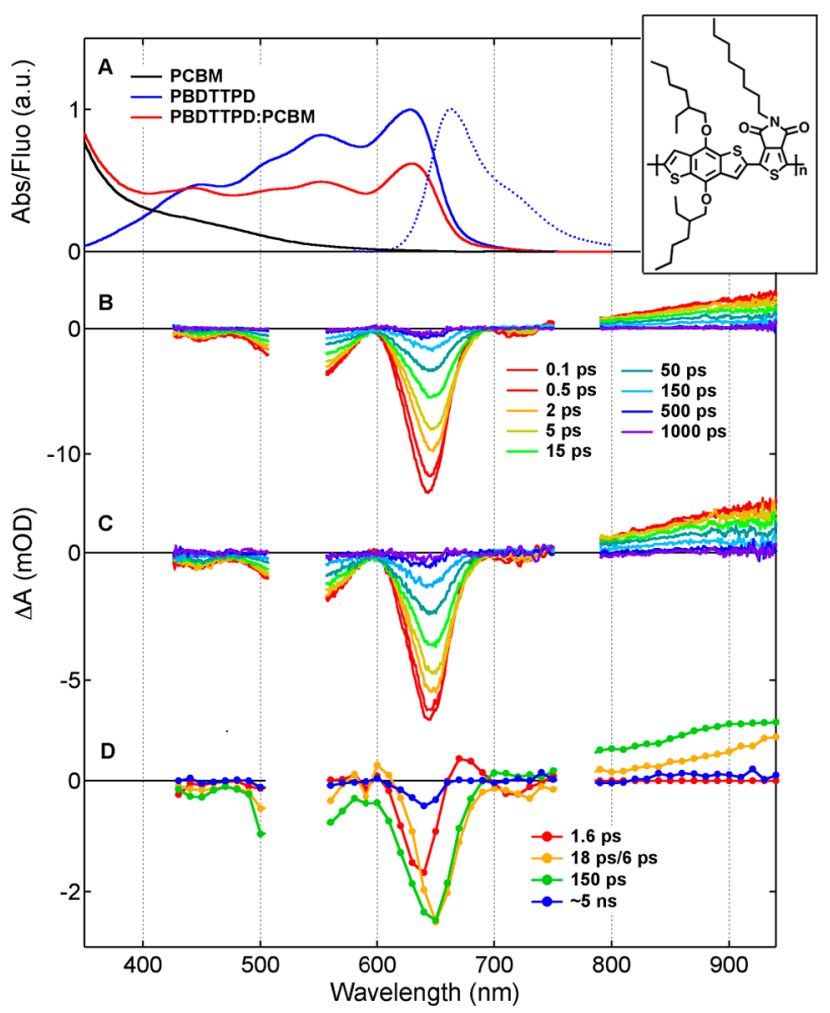

Figure 1. (A) Steady-state spectra of the investigated thin films and molecular structure of PBDTTPD. TA spectra of PBDTTPD with 530 $\mathrm{nm}$ excitation at (B) $16 \mu \mathrm{J} / \mathrm{cm}^{2}$ and (C) $8 \mu \mathrm{J} / \mathrm{cm}^{2}$. (D) Amplitude spectra resulting from the global analysis of time profiles recorded at 8 $\mu \mathrm{J} / \mathrm{cm}^{2}$.

polymer features are clearly evident in the absorption spectrum, together with the PCBM signature mainly below $500 \mathrm{~nm}$.

Femtosecond-resolved TA spectra were recorded with 530 $\mathrm{nm}$ excitation at pump fluences ranging from 4 to $40 \mu \mathrm{J} / \mathrm{cm}^{2}$. The spectra of pristine PBDTTPD film are shown for 8 and 16 $\mu \mathrm{J} / \mathrm{cm}^{2}$ in Figure $1 \mathrm{~B}-\mathrm{C}$, with selected time profiles at $8 \mu \mathrm{J} / \mathrm{cm}^{2}$ in Figure 2A. The blue side of the negative band with maxima around 445, 530, and $645 \mathrm{~nm}$ is assigned to the ground state bleach (GSB), based on its similarity with the steady-state absorption spectrum. The $645 \mathrm{~nm}$ TA peak is more red-shifted than the absorption, due to an overlap with the negative stimulated emission (SE) band around $660 \mathrm{~nm}$, at a similar position as the steady-state fluorescence. The weak negative signal in the $720 \mathrm{~nm}$ region is the second vibronic shoulder of the SE. At early time delays, the GSB/SE features around 645 and $720 \mathrm{~nm}$ show a clear $\sim 7 \mathrm{~nm}$ red shift caused by excited state relaxation (Figure 1B-C), which reflects, for example, as a fast decay at $640 \mathrm{~nm}$ and rise at $670 \mathrm{~nm}$ in the dynamics (Figure 2A). For conjugated polymers, relaxation typically includes localization of the photoexcitation, geometrical rearrangements (less important for PBDTTPD), ${ }^{8}$ and excitation energy transfer to longer polymer chain segments with lower energy. ${ }^{22-24}$ Changes in the vibronic structure during this relaxation might also be responsible for the rapid $(<10 \mathrm{ps})$ disappearance of the $720 \mathrm{~nm} \mathrm{SE}$, although the singlet excited state lives much longer. ${ }^{24}$ Finally, there is a broad positive TA band above $740 \mathrm{~nm}$, which does not show significant spectral dynamics and which we attribute predominantly to singlet excited state absorption (ESA) of PBDTTPD, because the lifetime of the $850 \mathrm{~nm}$ signal follows the one of the
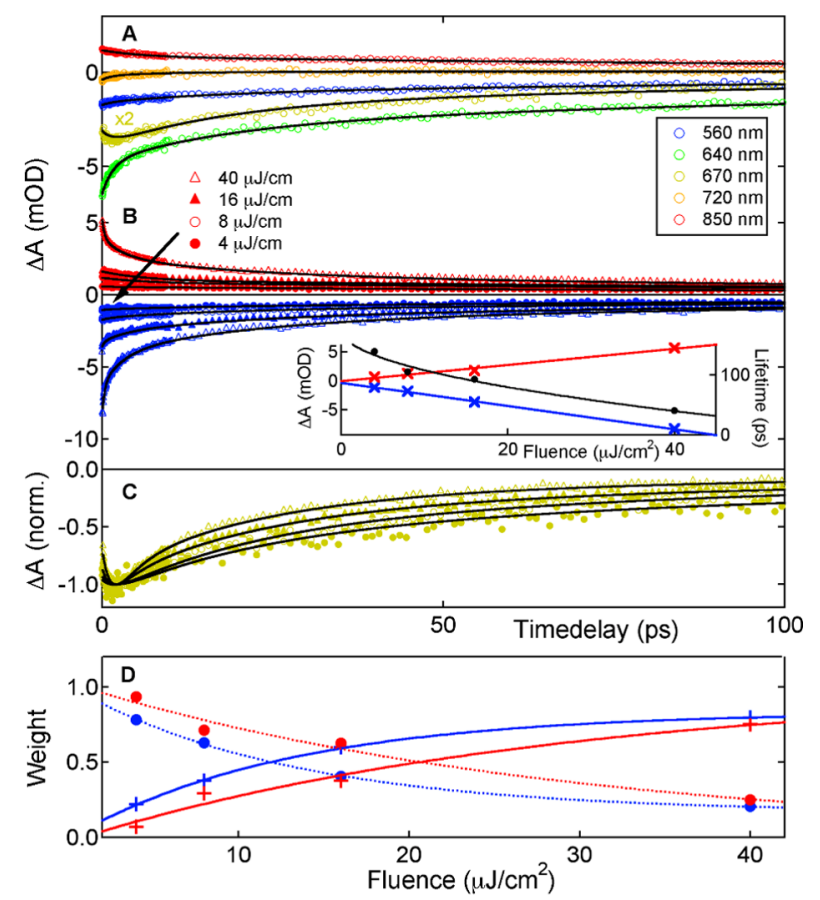

Figure 2. (A) TA time profiles of PBDTTPD with $530 \mathrm{~nm}$ excitation at $8 \mu \mathrm{J} / \mathrm{cm}^{2}$. (B) Time profiles at $560 \mathrm{~nm}$ (blue) and $850 \mathrm{~nm}$ (red) at various fluences. Inset: Initial TA intensity at the two wavelengths and average lifetime at $850 \mathrm{~nm}$ (black) as a function of fluence. (C) Normalized time profiles at $670 \mathrm{~nm}$ (dark yellow) at various fluences. (D) Summed weight of the short $\left(<22 \mathrm{ps}=\tau_{1}+\tau_{2}+\tau_{3}\right.$, + solid $)$ and long components $\left(150 \mathrm{ps}, \sim 5 \mathrm{~ns}=\tau_{4}+\tau_{5}\right.$, dotted) at $560 \mathrm{~nm}$ (blue) and $850 \mathrm{~nm}$ (red) as a function of fluence, solid lines are guide to the eye.

SE at $670 \mathrm{~nm}$ quite closely (Figure 2A, discussion below). As charges have been shown to exist in other pristine donoracceptor copolymers, especially at short time delays, ${ }^{25-27}$ we cannot exclude a weak contribution of charge absorption in the broad positive band.

Time profiles taken every $10 \mathrm{~nm}$ from TA spectra of PBDTTPD at $8 \mu \mathrm{J} / \mathrm{cm}^{2}$ were analyzed globally using the sum of exponential functions. The amplitude spectra (from the preexponential factors associated with the time constants) are shown in Figure 1D. Multiexponential global analysis gives an excellent idea of the time scales of photophysical processes too entangled to be reasonably reproduced by a kinetic model. ${ }^{28-31}$ Fast decay of the positive ESA signature is mirrored by decay of the GSB and SE features, indicating return of the excited singlet population to the ground state (Figure $2 \mathrm{~A}$ ). This is associated with a 6 ps time constant in the $>750 \mathrm{~nm}$ region (ESA), which has to be replaced by two 1.6 and 18 ps components in the $<750 \mathrm{~nm}$ range (GSB/SE), in order to account for the spectral shifts (relaxation) temporally mixed with singlet excited state decay (Table 1). The shape of the 1.6 and 18 ps amplitude spectra reflects the entanglement of the two processes (Figure 1D). For example, the blue side of the $645 \mathrm{~nm} \mathrm{GSB} / \mathrm{SE}$ peak decreases mainly with the shorter time constant, and the red side decreases with the longer component, due to parallel decay and red shift of the band. Further decay at all TA wavelengths then occurs with a 150 ps time constant (Table 1). According to the amplitude spectrum, it involves again the ESA ( $>750$ $\mathrm{nm}), \mathrm{SE}$ (around $660 \mathrm{~nm})$, and GSB $(<660 \mathrm{~nm})$, so that we assign it to decay from the relaxed singlet excited state to the ground state (Figure 1D). Finally, there is also a weak $\sim 5 \mathrm{~ns}$ 
Table 1. Time Constants (and Relative Amplitudes) from Global Analysis of the TA Time Profiles

\begin{tabular}{|c|c|c|c|c|c|c|c|}
\hline sample & $\lambda(\mathrm{nm})$ & fluence $\left(\mu \mathrm{J} / \mathrm{cm}^{2}\right)$ & $\tau_{1}(\mathrm{ps})$ & $\tau_{2}(\mathrm{ps})$ & $\tau_{3}(\mathrm{ps})$ & $\tau_{4}(\mathrm{ps})$ & $\tau_{5}(\mathrm{~ns})$ \\
\hline \multirow[t]{8}{*}{ PBDTTPD } & 560 & 4 & & $1.6(0.04)$ & $18.0(0.18)$ & $150(0.61)$ & $5(0.17)$ \\
\hline & & 8 & & $1.6(0.08)$ & $18.0(0.29)$ & $150(0.56)$ & $5(0.07)$ \\
\hline & & 16 & $0.35(0.08)$ & $3.1(0.18)$ & $22.2(0.34)$ & $150(0.34)$ & $5(0.06)$ \\
\hline & & 40 & $0.35(0.27)$ & $3.1(0.25)$ & $22.2(0.27)$ & $150(0.19)$ & $5(0.02)$ \\
\hline & 850 & 4 & & & $6.0(0.07)$ & $150(0.82)$ & $5(0.11)$ \\
\hline & & 8 & & & $6.0(0.29)$ & $150(0.64)$ & $5(0.07)$ \\
\hline & & 16 & & & $6.0(0.38)$ & $150(0.57)$ & $5(0.06)$ \\
\hline & & 40 & $0.35(0.23)$ & $3.1(0.26)$ & $22.2(0.26)$ & $150(0.21)$ & $5(0.03)$ \\
\hline \multirow[t]{8}{*}{ PBDTTPD:PCBM } & 640 & 4 & $0.94(0.31)$ & & & long (0.69) & \\
\hline & & 8 & $0.94(0.34)$ & & $190(0.14)$ & long (0.52) & \\
\hline & & 16 & $0.94(0.43)$ & & $190(0.21)$ & long (0.36) & \\
\hline & & 40 & $0.46(0.42)$ & $6.0(0.16)$ & $190(0.20)$ & long (0.22) & \\
\hline & 880 & 4 & & & $190(0.18)$ & long (0.82) & \\
\hline & & 8 & $0.94(0.04)$ & & $190(0.41)$ & long (0.55) & \\
\hline & & 16 & $0.94(0.21)$ & & $190(0.38)$ & long (0.41) & \\
\hline & & 40 & $0.46(0.13)$ & $6.0(0.24)$ & $190(0.37)$ & long (0.26) & \\
\hline
\end{tabular}

contribution to the TA dynamics (possibly longer, but the $1 \mathrm{~ns}$ experimental window does not allow a precise fit), evidencing a small population of long-lived triplet or charged polaron states, commonly observed in conjugated polymer films. ${ }^{10,32}$ It is not related to the singlet excited state, since the SE around $660 \mathrm{~nm}$ has vanished after 500 ps (only GSB at $635 \mathrm{~nm}$ is left, Figure 1B-D).

At excitation densities exceeding $10^{17}$ photons $/ \mathrm{cm}^{3}$, singlet excited species in conjugated polymer films are close enough to encounter and to undergo singlet exciton-exciton annihilation (EEA), which promotes one partner to the ground state and the other to a higher excited state. ${ }^{32-37}$ By assuming $\sim 90 \mathrm{~nm}$ film thickness for the used processing conditions and absorbance, ${ }^{3,6}$ the excitation density in our experiments ranged from $6 \times 10^{17}$ to $6 \times 10^{18}$ photons $/ \mathrm{cm}^{3}$ (Table S1, Supporting Information), so that EEA can be expected. Indeed, the average singlet excited state lifetime, estimated from the weighted average of all time constants except the $\sim 5 \mathrm{~ns}$ one at $850 \mathrm{~nm}$, decreases with square root dependence on fluence (inset of Figure $2 \mathrm{~B})$. There is faster decay of the ESA $(850 \mathrm{~nm})$ and GSB $(560 \mathrm{~nm})$ with increasing laser power (Figure 2B, more wavelengths in Figure S1, Supporting Information). Examination of Table 1 reveals that this is caused by increased weight of the short components $(6$ ps $>750 \mathrm{~nm}, 1.6$ and 18.0 ps $<750$ $\mathrm{nm}$ ), while the relative amplitude of the long (150 ps, $\sim 5 \mathrm{~ns}$ ) components decreases (illustrated in Figure 2D). We note that the short decay dynamics become more multiphasic at higher fluence, since EEA is diffusion-controlled and has a timedependent rate (faster before relaxation). ${ }^{33,35}$

The EEA mechanism (involvement of the singlet excited state) is further confirmed by the faster decay of the SE at 670 $\mathrm{nm}$ (normalized curves in Figure 2C), although quantification of the decay at this wavelength is hindered by the initial rise (relaxation). Intensity-induced loss of excited state population faster than the experimental time resolution can be excluded, since the initial TA intensities scale linearly with fluence (inset of Figure 2B). Although enhanced charge formation caused by EEA has been demonstrated in other conjugated polymers, ${ }^{32,35,37}$ this cannot be confirmed in PBDTTPD due to the similar shape of the TA spectra at different fluences (Figure S2, Supporting Information), and to the decrease of the $\sim 5 \mathrm{~ns}$ component (possibly polarons) at higher pump power. In conclusion, the ground state recovery on the $<22$ ps time scale is caused by singlet annihilation in pulsed laser excitation conditions, while the 150 ps component represents the "natural" lifetime of the relaxed PBDTTPD singlet excited state as expected under solar irradiation. The rate of EEA strongly depends on morphology, ${ }^{36}$ and its fast nature in PBDTTPD film suggests rather efficient diffusion of the singlet excited species.

The TA spectra of PBDTTPD:PCBM (mostly the polymer and not fullerene is excited at $530 \mathrm{~nm}$ ) reveal distinctly different and more long-lived features compared to the ones of the pristine polymer (Figure 3A-C). The GSB is still present below $660 \mathrm{~nm}$, but the SE $(\sim 660 \mathrm{~nm}, 720 \mathrm{~nm})$ is replaced by a relatively flat positive band that extends from 660 to $950 \mathrm{~nm}$ and has a peak at $880 \mathrm{~nm}$. This points to efficient quenching of the PBDTTPD excited state by CS between the polymer and the fullerene. We assign the new absorption signature to the positive polaron of PBDTTPD (either bound in an interfacial charge transfer (CT) state or free, as discussed below). A similar flat band extending next to the GSB with a peak at lower wavelengths has been observed in blends of other donoracceptor copolymers with PCBM, and was also assigned to the charged polymer. ${ }^{9,17}$ The anion of PCBM is known to absorb in the $1030 \mathrm{~nm}$ region, and is thus outside our measuring window. ${ }^{13}$

It has often been reported that CS occurs quantitatively in less that $100 \mathrm{fs}$ in polymer:fullerene bulk heterojunctions. ${ }^{14,38}$ Slower quenching (a few picoseconds) has been observed for blends with optimized morphology and larger polymer domains, implying increased distance of polymer excited species from the fullerene interface., ${ }^{9,11,12}$ In the case of PBDTTPD:PCBM, the polaron signature is already present at the earliest measurable time delay (Figure $3 \mathrm{~B}-\mathrm{C}$ ), confirming that part of the CS occurs within the 100 fs time resolution. Nevertheless, the early TA spectra in the blend show also features of the PBDTTPD singlet excited state, such as the SE around 660 and $720 \mathrm{~nm}$ or the ESA above $900 \mathrm{~nm}$, which disappear within about 2 ps (dynamics in Figure 4A). Global analysis yields a 0.94 ps time constant associated with this slower CS component. The shape of the associated amplitude spectrum clearly shows the decay of the SE and ESA signatures, as well the rise of the polaron band as an indent around $880 \mathrm{~nm}$ (Figure 3D, $8 \mu \mathrm{J} / \mathrm{cm}^{2}$ ). Although the quenching in the blend masks any signs of spectral relaxation in the SE, we infer from 


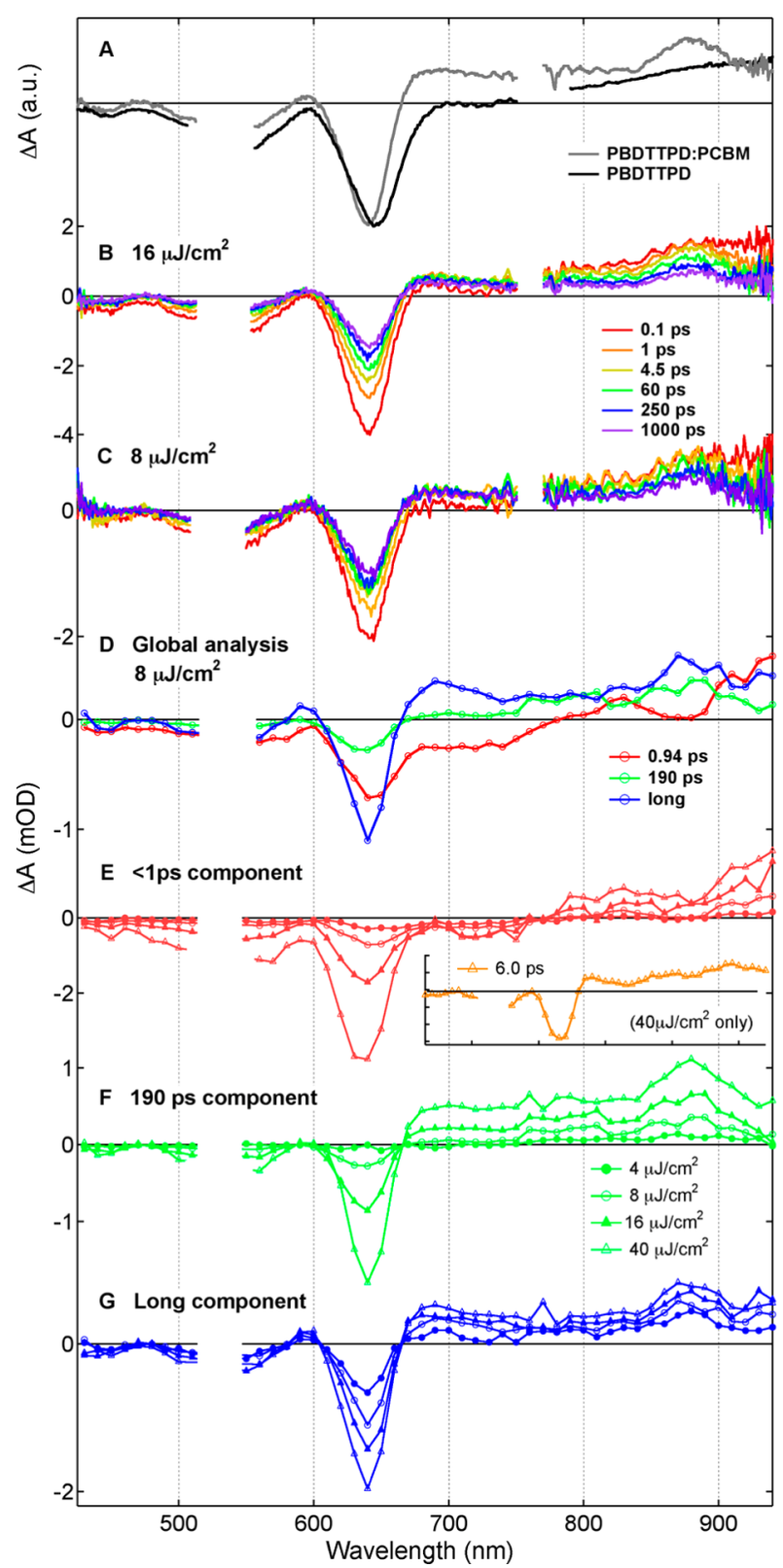

Figure 3. (A) TA spectra of PBDTTPD and PBDTTPD:PCBM 10 ps after excitation at $530 \mathrm{~nm}$ with $40 \mu \mathrm{J} / \mathrm{cm}^{2}$. (B,C) TA spectra of PBDTTPD:PCBM at 16 and $8 \mu \mathrm{J} / \mathrm{cm}^{2}$. (D) Amplitude spectra resulting from global analysis of the PBDTTPD:PCBM time profiles recorded at $8 \mu \mathrm{J} / \mathrm{cm}^{2}$. (E-G) Amplitude spectra associated with each time constant at different fluences.

the time scale of this relaxation in the pure polymer that $\mathrm{CS}$ in PBDTTPD:PCBM ( $<100 \mathrm{fs}, 0.94 \mathrm{ps}$ ) is fast enough to occur before full relaxation of the singlet excited state.

CS should not lead to any ground state recovery, nevertheless there is decrease of the GSB signature on the 1 ps time scale and this is reflected in the 0.94 ps amplitude spectrum (Figure 3B-D). A possible reason could be the growth of an overlapping positive band upon CS, or a smaller bleach cross section implying stronger localization of the charges compared to the singlet excited state. ${ }^{11,18}$ However, comparison of the TA spectra at 8 and $16 \mu \mathrm{J} / \mathrm{cm}^{2}$ (Figure 3BC) reveals enhanced signal decay at the higher fluence (TA spectra, time profiles, and outcome of global analysis for all investigated intensities are shown in Figures S3-S6 and Table
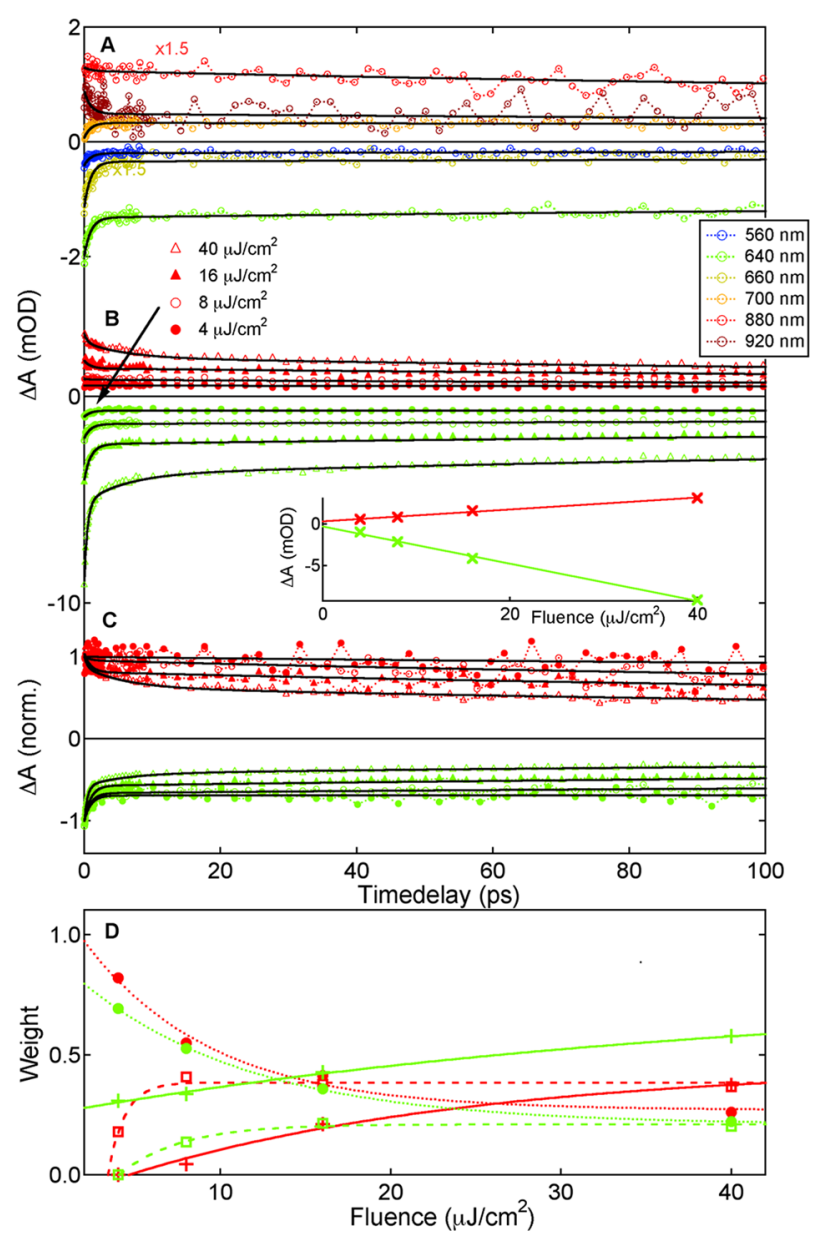

Figure 4. (A) TA time profiles of PBDTTPD:PCBM with $530 \mathrm{~nm}$ excitation at $8 \mu \mathrm{J} / \mathrm{cm}^{2}$. Time profiles at $640 \mathrm{~nm}$ (green) and $880 \mathrm{~nm}$ (red) at various fluences as measured (B) and normalized (C). Inset: Initial TA intensity at the two wavelengths (green/red) as a function of fluence. (D) Weight of the short ( $<7 \mathrm{ps}=\tau_{1}+\tau_{2}$, + solid), intermediate (190 ps $=\tau_{3}, \square$ dashed), and of the long components $\left(\tau_{4}\right.$, - dotted) at $640 \mathrm{~nm}$ (green) and $880 \mathrm{~nm}$ (red) as a function of fluence; solid lines are guide to the eye.

S2, Supporting Information). Indeed, the weight of the $0.94 \mathrm{ps}$ decay component at $640 \mathrm{~nm}(\mathrm{GSB} / \mathrm{SE})$ and at $880 \mathrm{~nm}$ (ESA and polaron absorption) increases with excitation fluence, and this time constant has to be replaced by two 0.46 and 6.0 ps ones at the highest power (Figure 4B-D, Table 1). This proves that a bimolecular loss mechanism occurs in parallel with the $\sim 1$ ps CS and is mainly responsible for the observed decrease in GSB. At $4 \mu \mathrm{J} / \mathrm{cm}^{2}$, the 0.94 ps component at $880 \mathrm{~nm}$ completely vanishes, indicating an "isosbestic point" between the neutral and charged signatures and almost negligible intensity-induced loss at our lowest fluence (Table 1).

To appreciate how the $0.94 \mathrm{ps}\left(0.46 \mathrm{ps}\right.$ at $\left.40 \mu \mathrm{J} / \mathrm{cm}^{2}\right)$ component evolves with fluence throughout the TA spectrum, the corresponding amplitude spectrum at different intensities is compared in Figure 3E. In all cases, there is decay of the singlet excited state signatures and rise of the polaron band, with no evidence for intensity-induced loss of charges. The decay of the singlet excited state is, however, increasingly accompanied by ground-state recovery at high fluence (see red shift of the $\sim 640$ $\mathrm{nm}$ feature due to increased GSB contribution). We conclude that the neutral excited state of the polymer decays in $\sim 1$ ps due to both CS with a fullerene and competing annihilation 
processes at high excitation densities. This can be EEA (as in the pristine polymer), but exciton-charge annihilation (ECA) can also occur, since the singlet excited state and charges coexist at short time delays. ECA has previously been reported in polymer:polymer and polymer:fullerene blends, and is even more efficient than EEA. ${ }^{18,20,39}$ The charges are preserved in this process (and promoted to a short-lived excited state), but the ultimate yield of charge carriers is reduced by loss of their neutral precursors to the ground state. Finally, a very important observation is that no annihilation occurs within the experimental time resolution (the initial TA amplitude scales linearly with fluence, inset of Figure $4 B$ ), while CS in $<100$ fs is efficient. Thus, EEA or ECA cannot compete with the prompt CS component, as diffusion does not seem to play a role on the ultrashort time scale.

There is also a global 190 ps decay component in the TA dynamics of PBDTTPD:PCBM (Table 1). According to the amplitude spectrum, it is associated with loss of charged species (see presence of the polaron signature) and with ground state recovery; there is no significant contribution of the singlet excited state (Figure 3D,F). Its relative weight at 640 and 880 $\mathrm{nm}$ does not significantly depend on fluence, except at the lowest laser power, where it almost vanishes (Figure 4D, Table 1). However, we note significant noise in the $4 \mu \mathrm{J} / \mathrm{cm}^{2}$ measurement on longer time scales, so that it is difficult to draw conclusions from a single wavelength. Judging from the amplitude spectrum, the 190 ps component is indeed present even at the lowest fluence (Figure 3F). Therefore, we suggest that monomolecular (fluence independent) charge recombination has a strong contribution to the 190 ps dynamics.

A possible mechanism is monomolecular trap-assisted recombination involving free charges, ${ }^{40}$ but this is typically slower. ${ }^{9,11}$ More probable is geminate charge recombination from an interfacial CT state. Time scales of hundreds of picoseconds to nanoseconds have been reported for this process in various polymers. ${ }^{9-11,13,17,41} \mathrm{We}$ are not able to distinguish charges that are bound in the CT state from free ones using the broad polaron absorption band (similar spectral signatures have also been noted in TA studies of some other polymers). ${ }^{9,10}$ On the basis of a model by Laquai et al., ${ }^{9-11}$ both are expected even at the earliest time delays, since they are instantaneously generated and do not interconvert. A recent pump-push photoconductivity experiment confirms that free charge carriers are generated from "hot" delocalized states populated directly following $\mathrm{CS}^{42}$ In photovoltaically highly efficient systems, only a small fraction of charges relaxes into the interfacial CT state and recombines geminately. The intensity-dependent part of the 190 ps time constant in PBDTTPD:PCBM might be explained by bimolecular recombination of free charges. Even if a much slower rate is expected based on reports about other polymers, ${ }^{10,19,41}$ and low hole mobility in PBDTTPD $\left(1.6 \times 10^{-4} \mathrm{~cm}^{2} /(\mathrm{V} \mathrm{s})\right){ }^{6}$ we hypothesize that the concentration of free charges at fluences as high as $40 \mu \mathrm{J} / \mathrm{cm}^{2}$ is sufficient to allow bimolecular encounter without large scale diffusion. In support of this, we observe an additional 6 ps time constant only at the highest laser power (Table 1), with an amplitude spectrum that suggests fast intensity-induced recombination of charge carriers (inset of Figure 3E).

At long time delays, there is a plateau at all wavelengths of the PBDTTPD:PCBM TA spectra, which is assigned to longlived (mainly free) polarons with a spectral signature characterized by the amplitude spectrum in Figure 3D,G. In the quasi absence of intensity-induced loss, we roughly estimate their yield to $>80 \%$ (at $880 \mathrm{~nm}, 4 \mu \mathrm{J} / \mathrm{cm}^{2}$, Table 1), indicating a relatively low fraction of geminately recombining charges formed in the CT state. With higher fluence, the weight of the long component is strongly reduced, in excellent agreement between the $640 \mathrm{~nm}$ GSB and $880 \mathrm{~nm}$ polaron dynamics (Figure 4C,D). Our work suggests that the lower yield of longlived charge carriers at high intensity is mainly caused by annihilation processes of the neutral excited state, and possibly some bimolecular charge recombination at very high fluence.

In conclusion, detailed analysis of the intensity dependent TA spectra of PBDTTPD and PBDTTPD:PCBM thin films has led to a very clear picture of the excited state dynamics occurring in this solar cell material. The relaxed singlet excited state of the pristine polymer decays to the ground state with a 150 ps time constant; only a small fraction of long-lived polaron or triplet states is populated. At high fluence, there is additional multiphasic EEA occurring on the $<22$ ps time scale. In the presence of fullerene, singlet excited species of the polymer formed close to a fullerene interface undergo charge separation in $<100 \mathrm{fs}$. In the investigated fluence range, this process does not compete with exciton annihilation (no ultrafast diffusive encounter). On the other hand, we identified a slower $\sim 1$ ps quenching component that strongly competes with EEA and ECA at higher pump intensities, leading to a decrease of charge carrier yield with fluence. We were able to reduce the fluence to a point were the intensity-induced loss becomes negligible. In this case, $>80 \%$ of charge carriers survive beyond $1 \mathrm{~ns}$, the rest is lost on the 200 ps time scale, most probably due to geminate recombination.

\section{ASSOCIATED CONTENT}

\section{Supporting Information}

Experimental details, determination of excitation density, and additional figures and tables as mentioned in the text. This material is available free of charge via the Internet at http:// pubs.acs.org.

\section{AUTHOR INFORMATION}

\section{Corresponding Author}

*E-mail: natalie.banerji@epfl.ch.

Notes

The authors declare no competing financial interest.

\section{ACKNOWLEDGMENTS}

N.B. thanks the Swiss National Science Foundation for funding through the Ambizione Fellowship PZ00P2_136853.

\section{REFERENCES}

(1) Heeger, A. J.; Sariciftci, N. S.; Namdas, E. B. Semiconducting and Metallic Polymers; Oxford University Press: Oxford, UK, 2010.

(2) Thompson, B. C.; Frechet, J. M. J. Polymer-Fullerene Composite Solar Cells. Angew. Chem., Int. Ed. Engl. 2008, 47, 58-77.

(3) Zou, Y.; Najari, A.; Berrouard, P.; Beaupre, S.; Reda, A. B.; Tao, Y.; Leclerc, M. A Thieno[3,4-c]pyrrole-4,6-dione-Based Copolymer for Efficient Solar Cells. J. Am. Chem. Soc. 2010, 132, 5330-5331.

(4) Zhang, Y.; Hau, S. K.; Yip, H.-L.; Sun, Y.; Acton, O.; Jen, A. K. Y. Efficient Polymer Solar Cells Based on the Copolymers of Benzodithiophene and Thienopyrroledione. Chem. Mater. 2010, 22, 2696-2698.

(5) Piliego, C.; Holcombe, T. W.; Douglas, J. D.; Woo, C. H.; Beaujuge, P. M.; Frechet, J. M. J. Synthetic Control of Structural Order in N-Alkylthieno[3,4-c]pyrrole-4,6-dione-Based Polymers for Efficient Solar Cells. J. Am. Chem. Soc. 2010, 132, 7595-7597. 
(6) Aich, B. R.; Lu, J.; Beaupre, S.; Leclerc, M.; Tao, Y. Control of the Active Layer Nanomorphology by Using Co-additives Towards HighPerformance Bulk Heterojunction Solar Cells. Org. Electron. 2012, 13, $1736-1741$.

(7) Shuttle, C. G.; Treat, N. D.; Douglas, J. D.; Frechet, J. M. J.; Chabinyc, M. L. Deep Energetic Trap States in Organic Photovoltaic Devices. Adv. Energy Mater. 2012, 2, 111-119.

(8) Hwang, I.; Beaupre, S.; Leclerc, M.; Scholes, G. D. Ultrafast Relaxation of Charge-Transfer Excitons in Low-Bandgap Conjugated Copolymers. Chem. Sci. 2012, 3, 2270-2277.

(9) Etzold, F.; Howard, I. A.; Forler, N.; Cho, D. M.; Meister, M.; Mangold, H.; Shu, J.; Hansen, M. R.; Muellen, K.; Laquai, F. The Effect of Solvent Additives on Morphology and Excited-State Dynamics in PCPDTBT:PCBM Photovoltaic Blends. J. Am. Chem. Soc. 2012, 134, 10569-10583.

(10) Etzold, F.; Howard, I. A.; Mauer, R.; Meister, M.; Kim, T.-D.; Lee, K.-S.; Baek, N. S.; Laquai, F. Ultrafast Exciton Dissociation Followed by Nongeminate Charge Recombination in PCDTBT:PCBM Photovoltaic Blends. J. Am. Chem. Soc. 2011, 133, 9469-9479.

(11) Howard, I. A.; Mauer, R; Meister, M.; Laquai, F. Effect of Morphology on Ultrafast Free Carrier Generation in Polythiophene:Fullerene Organic Solar Cells. J. Am. Chem. Soc. 2010, 132, 1486614876.

(12) Marsh, R. A.; Hodgkiss, J. M.; Albert-Seifried, S.; Friend, R. H. Effect of Annealing on P3HT:PCBM Charge Transfer and Nanoscale Morphology Probed by Ultrafast Spectroscopy. Nano Lett. 2010, 10, 923-930.

(13) Guo, J. M.; Ohkita, H.; Benten, H.; Ito, S. Charge Generation and Recombination Dynamics in Poly(3-hexylthiophene)/Fullerene Blend Films with Different Regioregularities and Morphologies. J. Am. Chem. Soc. 2010, 132, 6154-6164.

(14) Tong, M. H.; Coates, N. E.; Moses, D.; Heeger, A. J.; Beaupre, S.; Leclerc, M. Charge Carrier Photogeneration and Decay Dynamics in the Poly(2,7-carbazole) Copolymer PCDTBT and in Bulk Heterojunction Composites with PC70BM. Phys. Rev. B 2010, 81, 125210 .

(15) Cook, S.; Katoh, R; Furube, A. Ultrafast Studies of Charge Generation in PCBM:P3HT Blend Films Following Excitation of the Fullerene PCBM. J. Phys. Chem. C 2009, 113, 2547-2552.

(16) Hwang, I. W.; Moses, D.; Heeger, A. J. Photoinduced Carrier Generation in P3HT/PCBM Bulk Heterojunction Materials. J. Phys. Chem. C 2008, 112, 4350-4354.

(17) De, S.; Pascher, T.; Maiti, M.; Jespersen, K. G.; Kesti, T.; Zhang, F. L.; Inganas, O.; Yartsev, A.; Sundstrom, V. Geminate Charge Recombination in Alternating Polyfluorene Copolymer/Fullerene Blends. J. Am. Chem. Soc. 2007, 129, 8466-8472.

(18) Hodgkiss, J. M.; Albert-Seifried, S.; Rao, A.; Barker, A. J.; Campbell, A. R.; Marsh, R. A.; Friend, R. H. Exciton-Charge Annihilation in Organic Semiconductor Films. Adv. Funct. Mater. 2012, 22, 1567-1577.

(19) Clarke, T. M.; Jamieson, F. C.; Durrant, J. R. Transient Absorption Studies of Bimolecular Recombination Dynamics in Polythiophene/Fullerene Blend Films. J. Phys. Chem. C 2009, 113, 20934-20941.

(20) Ferguson, A. J.; Kopidakis, N.; Shaheen, S. E.; Rumbles, G. Quenching of Excitons by Holes in Poly(3-hexylthiophene) Films. J. Phys. Chem. C 2008, 112, 9865-9871.

(21) Risko, C.; McGehee, M. D.; Bredas, J. L. A Quantum-Chemical Perspective into Low Optical-Gap Polymers for Highly-Efficient Organic Solar Cells. Chem. Sci. 2011, 2, 1200-1218.

(22) Wells, N. P.; Boudouris, B. W.; Hillmyer, M. A.; Blank, D. A. Intramolecular Exciton Relaxation and Migration Dynamics in Poly(3hexylthiophene). J. Phys. Chem. C 2007, 111, 15404-15414.

(23) Banerji, N.; Cowan, S.; Leclerc, M.; Vauthey, E.; Heeger, A. J. Exciton Formation, Relaxation and Decay in PCDTBT. J. Am. Chem. Soc. 2010, 132, 17459-17470.
(24) Banerji, N.; Cowan, S.; Vauthey, E.; Heeger, A. J. Ultrafast Relaxation of the Poly(3-hexylthiophene) Emission Spectrum. J. Phys. Chem. C 2011, 115, 9726-9739.

(25) Tautz, R.; Da Como, E.; Limmer, T.; Feldmann, J.; Egelhaaf, H.J.; von Hauff, E.; Lemaur, V.; Beljonne, D.; Yilmaz, S.; Dumsch, I.; et al. Structural Correlations in the Generation of Polaron Pairs in Low-Bandgap Polymers for Photovoltaics. Nat. Commun. 2012, 3, 970.

(26) Rolczynski, B. S.; Szarko, J. M.; Son, H. J.; Liang, Y. Y.; Yu, L. P.; Chen, L. X. Ultrafast Intramolecular Exciton Splitting Dynamics in Isolated Low-Band-Gap Polymers and Their Implication in Photovoltaic Materials Design. J. Am. Chem. Soc. 2012, 134, 4142-4152.

(27) Carsten, B.; Szarko, J. M.; Son, H. J.; Wang, W.; Lu, L. Y.; He, F.; Rolczynski, B. S.; Lou, S. J.; Chen, L. X.; Yu, L. P. Examining the Effect of the Dipole Moment on Charge Separation in DonorAcceptor Polymers for Organic Photovoltaic Applications. J. Am. Chem. Soc. 2011, 133, 20468-20475.

(28) Banerji, N.; Duvanel, G.; Perez-Velasco, A.; Maity, S.; Sakai, N.; Matile, S.; Vauthey, E. Excited-State Dynamics of Hybrid Multichromophoric Systems: Toward an Excitation Wavelength Control of the Charge Separation Pathways. J. Phys. Chem. A 2009, 113, 82028212.

(29) Fita, P.; Luzina, E.; Dziembowska, T.; Radzewicz, C.; Grabowska, A. Chemistry, Photophysics, and Ultrafast Kinetics of Two Structurally Related Schiff Bases Containing the Naphthalene or Quinoline Ring. J. Chem. Phys. 2006, 125.

(30) van Stokkum, I. H. M.; Larsen, D. S.; van Grondelle, R. Global and Target Analysis of Time-Resolved Spectra. Biochim. Biophys. Acta, Bioenerg. 2004, 1657, 82-104.

(31) Holzwarth, A. R. Data Analysis of Time-Resolved Measurements. In Biophysical Techniques in Photosynthesis; Amesz, J., Hoff, A. J., Eds.; Springer: Dordrecht, The Netherlands, 2004.

(32) Guo, J. M.; Ohkita, H.; Benten, H.; Ito, S. Near-IR Femtosecond Transient Absorption Spectroscopy of Ultrafast Polaron and Triplet Exciton Formation in Polythiophene Films with Different Regioregularities. J. Am. Chem. Soc. 2009, 131, 16869-16880.

(33) Cook, S.; Han, L. Y.; Furube, A.; Katoh, R. Singlet Annihilation in Films of Regioregular Poly(3-hexylthiophene): Estimates for Singlet Diffusion Lengths and the Correlation between Singlet Annihilation Rates and Spectral Relaxation. J. Phys. Chem. C 2010, 114, 1096210968.

(34) Lewis, A. J.; Ruseckas, A.; Gaudin, O. P. M.; Webster, G. R.; Burn, P. L.; Samuel, I. D. W. Singlet Exciton Diffusion in MEH-PPV Films Studied by Exciton-Exciton Annihilation. Org. Electron. 2006, 7, $452-456$.

(35) Martini, I. B.; Smith, A. D.; Schwartz, B. J. Exciton-Exciton Annihilation and the Production of Interchain Species in Conjugated Polymer Films: Comparing the Ultrafast Stimulated Emission and Photoluminescence Dynamics of MEH-PPV. Phys. Rev. B 2004, 69, 035204.

(36) Nguyen, T. Q.; Martini, I. B.; Liu, J.; Schwartz, B. J. Controlling Interchain Interactions in Conjugated Polymers: The Effects of Chain Morphology on Exciton-Exciton Annihilation and Aggregation in MEH-PPV Films. J. Phys. Chem. B 2000, 104, 237-255.

(37) Peckus, D.; Devizis, A.; Hertel, D.; Meerholz, K.; Gulbinas, V. Exciton Diffusion, Annihilation and Their Role in the Charge Carrier Generation in Fluorene Based Copolymers. Chem. Phys. 2012, 404, $42-47$.

(38) Brabec, C. J.; Zerza, G.; Cerullo, G.; De Silvestri, S.; Luzzati, S.; Hummelen, J. C.; Sariciftci, S. Tracing Photoinduced Electron Transfer Process in Conjugated Polymer/Fullerene Bulk Heterojunctions in Real Time. Chem. Phys. Lett. 2001, 340, 232-236.

(39) Howard, I. A.; Hodgkiss, J. M.; Zhang, X.; Kirov, K. R.; Bronstein, H. A.; Williams, C. K.; Friend, R. H.; Westenhoff, S.; Greenham, N. C. Charge Recombination and Exciton Annihilation Reactions in Conjugated Polymer Blends. J. Am. Chem. Soc. 2010, 132, $328-335$

(40) Cowan, S. R.; Leong, W. L.; Banerji, N.; Dennler, G.; Heeger, A. J. Identifying a Threshold Impurity Level for Organic Solar Cells: Enhanced First-Order Recombination Via Well-Defined PC84BM 
Traps in Organic Bulk Heterojunction Solar Cells. Adv. Funct. Mater.

2011, 21, 3083-3092.

(41) Massip, S.; Oberhumer, P. M.; Tu, G.; Albert-Seifried, S.; Huck, W. T. S.; Friend, R. H.; Greenham, N. C. Influence of Side Chains on Geminate and Bimolecular Recombination in Organic Solar Cells. J. Phys. Chem. C 2011, 115, 25046-25055.

(42) Bakulin, A. A.; Rao, A.; Pavelyev, V. G.; van Loosdrecht, P. H. M.; Pshenichnikov, M. S.; Niedzialek, D.; Cornil, J.; Beljonne, D.; Friend, R. H. The Role of Driving Energy and Delocalized States for Charge Separation in Organic Semiconductors. Science (Washington, DC, U. S.) 2012, 335, 1340-1344. 